\title{
Teen-Robot Interaction
}

\section{A Pilot Study of Engagement with a Low-fidelity Prototype}

\author{
Elin A. Björling \\ University of Washington \\ Seattle \\ USA \\ bjorling@uw.edu
}

\author{
Emma Rose \\ University of Washington \\ Tacoma \\ USA \\ ejrose@uw.edu
}

\author{
Rachel Ren \\ University of Washington \\ Seattle \\ USA \\ rachren@uw.edu
}

\begin{abstract}
Today's teens will most likely be the first generation to spend a lifetime living and interacting with both mechanical and social robots. Although human-robot interaction has been explored in children, adults, and seniors, examination of teen-robot interaction has been minimal. Using human-centered design, our team is developing a social robot to gather stress and mood data from teens in a public high school. As part of our preliminary design stage, we conducted a interaction pilot study in the wild to explore and capture teens' initial interactions with a lowfidelity social robot prototype. We observed strong engagement and expressions of empathy from teens during our qualitative, interaction studies.
\end{abstract}

ACM Reference format:

Elin A. Björling, Emma Rose, and Rachel Ren. 2018. Teen-Robot Interaction: A Pilot Study of Engagement with a Low-fidelity Prototype. In HRI'18 Companion: Conference on ACM/IEEE International Conference on Human-Robot Interaction, March 5-8, 2018, Chicago, IL, USA. ACM, NY, NY, USA, 2 pages. DOI: https://doi.org/10.1145/3173386.3177068

\section{KEYWORDS}

Teen-robot interaction, engagement, prototype

\section{INTRODUCTION}

Many of today's adolescents have been surrounded by technology since birth. And, their relationships with current technology are unlike previous generations as much of their communication is now digital [1]. Despite teens' strong relationship with technology, they remain a fairly underexplored population when it comes to Human-Robot Interaction. Our team is researching what aspects of robot design lead to teen engagement. In our brief report, the following study details findings from a series of qualitative, observational interaction

Permission to make digital or hard copies of part or all of this work for personal or classroom use is granted without fee provided that copies are not made or distributed for profit or commercial advantage and that copies bear this notice and the full citation on the first page. Copyrights for third-party components of this work must be honored. For all other uses, contact the Owner/Author.

HRI '18 Companion, March 5-8, 2018, Chicago, IL, USA.

(C) 2018 Copyright is held by the owner/author(s)

ACM ISBN 978-1-4503-5615-2/18/03.

DOI: https://doi.org/10.1145/3173386.3177068 studies with teens and low-fidelity social robot prototype in their high school setting.

\section{BACKGROUND}

\subsection{Project EMAR Exploring Teen-Robot Interaction}

Although teens are avid consumers and users of new technologies, the relationship between teens and robots has been minimally explored, e.g. [2], [3]. Our team is developing a social robot, called EMAR (Ecological Momentary Assessment Robot). Ecological Momentary Assessment has been found an effective approach to capturing longitudinal data from teens, specifically to capture teen stress [4]. Therefore, EMAR is intended to live in a high school to engage and interact with teens while gathering stress and mood data. The following brief is data from a preliminary pilot of a low-fidelity prototype, EMAR Version 2.

\section{METHODS}

Using Human-Centered Design [5] to explore teens' interactions with EMAR V2 (a preliminary design prototype), we conducted a series of qualitative, observational interaction studies to explore how teens interacted with our boxy, low-fidelity prototype. See figure 1a. These studies were conducted "in the wild" [6] to capture data within the human social context of a public high school and thus maintain the ecological validity. Participants were invited to interact with the prototype in small groups (3-4 students), each taking turns responding to the robot's questions about mood, energy, and stress levels. This research study was reviewed and approved by both our University's Institutional Review Board and the school district's research review committee.

\subsection{EMAR V2 Prototype}

EMAR V2, built for this study, is a portable robot prototype that can interact in real-time with participants, engaging in a dialog and recording responses, see fig. 1, a. EMAR V2 has a minimal script which asks three questions about stress, mood, and energy. Respondents use a visual analogue scale to respond although data is not captured. During the interactive dialog, EMAR V2's eyes change their pattern to correspond with the answers that are played in audio. 


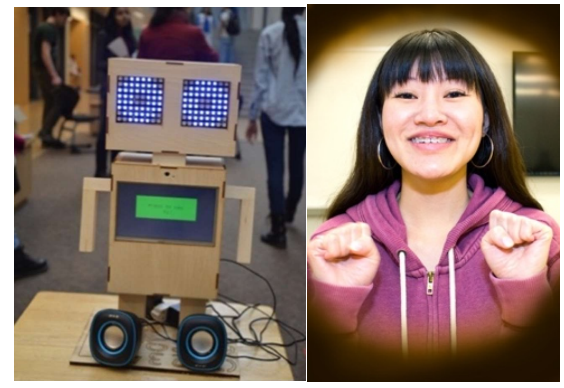

Figure 1: a. EMAR V2 Prototype (left), b. View of student researcher from internal camera (right)

\subsection{Data Analysis}

A total of 103 minutes of digital video data were captured and analyzed using thematic analysis [7].

\section{RESULTS}

\subsection{Participants}

The team conducted four studies at four high schools with a total of 45 participants. The schools were all located in a major urban area in the Pacific Northwest. In total, 27 males and 18 females participated in our study. Ages ranged from 14 to 18 years.

\subsection{Engagement}

The main theme that emerged from the qualitative, observational interaction study data was strong engagement with the lowfidelity prototype. We saw almost all participants with engaged and expressive faces while interacting with the prototype. One student said, "I could tell that EMAR was a machine, but EMAR was also so cute, that it was like, I could keep doing the EMAR thing over and over again, it didn't really get boring because EMAR is just kind of adorable." (Female, 16). Teens also expressed disappointment when their interaction session was over. A few teens asked if EMAR could stay with them at school. Another interesting finding was empathy. Participants showed empathy toward the prototype and toward one another during interaction sessions. Both users and bystanders mimicked EMAR's mood, or expressed emotion in relation to EMAR's responses. In one case, an older male teen physically hugged the prototype when his session ended saying, "Aww, goodbye, buddy." Teens found EMAR to be very "cute" and "likable" perhaps increasing their desire for interaction. As one participant expressed, "It's so cute you don't want to say no to it, you want to like talk to it, like, here let me explain my problems to you" (male, age 17).

\section{DISCUSSION}

The goal of this study was to explore the possibility of a social robot to support the mental health of teens. As detailed in the findings from the study, 45 teens across four schools, interacted with an early, low fidelity prototype to explore the potential for social robots within a school context. Key findings include strong engagement in the low fidelity prototype suggesting the promise of social robots in this context.

\subsection{Teen-Robot Interaction}

Surrounded by technology since birth, teens are most likely to have long-lasting relationships with high-level technology, including robots in their future work, education, and home settings. Engagement is a key factor in developing a successful social robot. Our finding that teens showed strong engagement with EMAR V2 was promising.

\subsection{LIMITATIONS}

Although a qualitative study, our small sample size limits the transference of this data to other populations and environments. Additionally, in this early stage of our research, it is not possible to understand whether the interaction and engagement we are seeing with our prototype is merely due to its novelty. Further research is required.

\subsection{CONCLUSION AND FUTURE RESEARCH}

Our preliminary interaction studies provided strong evidence for both teen's engagement and their emotional response with a social robot prototype. In addition, this preliminary study is evidence that even a low-fidelity prototype allowed for strong engagement and rich observational data. In the future, we plan to continue to engage teens in the human-centered design process and explore their needs in a social robot through participatory design, iterative prototyping and eventually, a longitudinal study of in-school interactions.

\section{ACKNOWLEDGMENTS}

Thank you to all the teens who participated in this research and to the wonderful graduate and undergraduate students who engaged in Project EMAR. This work is supported in part by the National Science Foundation under grant no. NRI-1734100.

\section{REFERENCES}

[1] A. Lenhart, "Teens, Social Media," Pew Research Center: Internet and Technology, 2015.

[2] N. Martelaro, V. C. Nneji, W. Ju, and P. Hinds, "Tell me more: Designing HRI to encourage more trust, disclosure, and companionship," ACM/IEEE Int. Conf. Human-Robot Interact., vol. 2016-April, pp. 181-188, 2016.

[3] E. J. Rose and E. A. Björling, "Designing for engagement," in Proceedings of the 35th ACM International Conference on the Design of Communication - SIGDOC '17, 2017, pp. 1-10.

[4] J. D. Runyan and E. G. Steinke, "Virtues, ecological momentary assessment/intervention and smartphone technology.," Front. Psychol., vol. 6, p. 481, 2015.

[5] J. Giacomin, "What is human centred design?," Des. J., vol. 17, no. 4, pp. 606-623, 2014.

[6] Y. Rogers and P. Marshall, "Research in the Wild," Synth. Lect. Human-Centered Informatics, vol. 10, no. 3, p. i-97, Apr. 2017.

[7] J. Fereday and E. Muir-Cochrane, "Demonstrating Rigor Using Thematic Analysis: A Hybrid Approach of Inductive and Deductive Coding and Theme Development," Int. J. Qual. Methods, vol. 5, no. 1, pp. 80-92, Mar. 2006. 